\title{
Gestão da Assistência Farmacêutica: Análise da situação de alguns municípios.
}

\section{Pharmaceutical Services Management: Analysis of the situation in some municipalities.}

\section{Gestión de los Servicios Farmacéuticos: Situación en algunos municipios brasileños.}

Fabiola Sulpino VIEIRA ${ }^{1}$

Paola ZUCCHI ${ }^{2}$

RESUMO: Os objetivos deste trabalho são descrever e analisar a gestão da assistência farmacêutica em alguns municípios, produzindo mais evidências sobre problemas que podem comprometer o acesso a medicamentos no Sistema Único de Saúde - SUS. Realizou-se pesquisa exploratória, transversal, elaborando-se questionário com questões fechadas sobre a gestão da assistência farmacêutica. $\mathrm{O}$ tamanho da amostra foi definido em 431 municípios, selecionados por amostragem aleatória estratificada. Dos 431 municípios, apenas 36 responderam o questionário (8,4\%). Destes, $44,4 \%(n=16)$ informaram ter a assistência farmacêutica formalizada, das quais $93,4 \%(n=15)$ contam com pelo menos um farmacêutico. Dos 36 municípios, 97\% $(\mathrm{n}=35)$ informaram ter lista de medicamentos para dispensação à população. $\mathrm{O}$ controle de estoque foi considerado adequado em 26 deles (72,2\%). Estão cumprindo determinação judicial para o fornecimento de medicamentos 14 (38,9\%) municípios e em 13 deles (36\%) todas as Unidades Básicas de Saúde - UBS contam com farmacêutico. Os resultados reforçam a necessidade do SUS de avançar no fortalecimento da gestão da assistência farmacêutica a fim de garantir o acesso a medicamentos e a efetividade das ações de saúde. Palavras chave: Assistência Farmacêutica. Sistema Único de Saúde. Gastos em Saúde. Acesso aos Serviços de Saúde. Gestão em Saúde.

1 Possui graduação em Farmácia-Bioquímica pela Universidade Estadual Paulista Júlio de Mesquita Filho - UNESP (1997), Mestrado em Química Orgânica pela Universidade Federal de São Carlos - UFSCar (1999), Mestrado Profissionalizante em Economia da Saúde pela Universidade Federal de São Paulo - UNIFESP (2006) e Doutorado em Saúde Coletiva pela UNIFESP (2010). É Especialista em Gestão Pública pela Fundação Escola de Sociologia e Política de São Paulo - FESPSP (2005) e trabalha como Especialista em Políticas Públicas e Gestão Governamental no Ministério da Saúde.

2 Possui graduação em Medicina pela Universidade Federal de São Paulo (1986), mestrado em Administração de Empresas pela Escola de Administração de Empresas de São Paulo - Fundação Getúlio Vargas (1995); doutorado em Saúde Pública pela Universidade de São Paulo - Faculdade de Saúde Pública (2002) e Livre Docente pela Universidade Federal de São Paulo - Centro Interdepartamental de Economia da Saúde - CRIDESUNIFESP. Atualmente é Professora Afiliada - Modalidade Pesquisa da Universidade Federal de São Paulo, Diretora da Companhia Paulista de Reabilitação - REABILITA e Vice Diretora do Centro Interdepartamental de Economia da Saúde da Universidade Federal de São Paulo - CRIDESUNIFESP. Tem experiência na área de Administração, com ênfase em Administração de Empresas, atuando principalmente nos seguintes temas: Economia da Saúde, Marketing em Saúde e Gestão e Administração em Saúde. 
SUMMARY: The objectives of this paper are to describe and to analyze the pharmaceutical services management in some Brazilian municipalities, producing more evidence about problems that may hamper access to medicines in Unified Health System - SUS. We conducted an exploratory, crosssectional research, drawing up a questionnaire with closed questions on pharmaceutical services management. The sample size was defined in 431 municipalities, selected by stratified random sampling. Only 36 municipalities of 431 answered the questionnaire (8.4\%). Of these, 44.4\% (n $=16)$ reported having formalized pharmaceutical services, of which $93.4 \%(n=15)$ have at least one pharmacist. Of the 36 municipalities, 97\% $(n=35)$ reported having drug list for dispensing to citizens and inventory control was considered suitable in 26 of them (72.2\%). Fourteen (38.9\%) municipalities are complying with court order for drug supply and 13 (36\%) have a pharmacist working at Basic Health Units - UBS. In conclusion, the SUS needs to advance much in order to ensure access to medicines and health effectiveness through efficient pharmaceutical services management. Key words: Pharmaceutical Services. Unified Health System. Health Expenditures. Health Services Accessibility. Health Management.

RESUMEN: Nuestro objetivo es describir y analizar la gestión de los servivios farmacéuticos en algunos municipios, para producir más proebas acerca de los problemas que podrían poner en peligro el acceso a los medicamentos en el Sistema Nacional de Salud (SUS). Hemos llevado a cabo um estudio exploratorio y transversal. Se elaboró un cuestionario con preguntas cerradas sobre la gestión de los servicios farmacéuticos. El tamaño de la muestra se definió en 431 municipios, seleccionados por muestreo aleatorio estratificado. De los 431 municipios, sólo 36 respondieron al cuestionario $(8,4 \%)$. De éstos, el 44,4\% $(n=16)$ reportaron tener uma estructura formal para gestión de los servicios farmacéuticos, de los cuales $93,4 \%(n=15)$ tenen al menos un farmacéutico. De los 36 municipios, 97\% $(\mathrm{n}=35)$ informó tener una lista de medicamentos para dispensar al público. El control de inventarios se consideró adecuado en 26 de ellos (72,2\%). Están cumpliendo con una orden judicial para el suministro de medicamentos 14 (38,9\%) de los municipios y en 13 de ellos (36\%) todas las Unidades Básicas de Salud tienen un farmacéutico. Los resultados refuerzan la necesidad de fortalecer el SUS en la gestión de los servicios farmacéuticos para garantizar el acceso a los medicamentos y la eficacia de las acciones de salud.

Palavras-clave: Servicios farmacêuticos. Sistema Nacional de Salud. Gastos en salud. Acceso a los servicios de salud. Gestión en salud.

\section{INTRODUÇÃO}

A assistência farmacêutica ganhou projeção na última década em decorrência, especialmente, do aumento no número de ações judiciais que determinam aos gestores do Sistema Único de Saúde (SUS) o fornecimento de medicamentos a cidadãos. O acesso a medicamentos e a integralidade da assistência terapêutica passaram a ser debatidos com maior freqüência, explicitando-se que para sua concretização também é preciso que atividades relativas ao campo da gestão sejam desenvolvidas satisfatoriamente. 
De acordo com a Organização Mundial da Saúde (OMS), o acesso a medicamentos essenciais depende de quatro fatores, quais sejam: seleção racional, financiamento sustentável, preços acessíveis e sistema de saúde de confiança. ${ }^{1}$ Dessa forma, está implícita na necessidade de se dispor de sistema de saúde de confiança, a boa gestão das ações e serviços, incluindo a assistência farmacêutica.

A formalização de um primeiro conceito de assistência farmacêutica no Brasil se deu com a publicação da Política Nacional de Medicamentos, sendo a assistência farmacêutica definida como um grupo de atividades relacionadas com o medicamento cujo objetivo é apoiar as ações de saúde em uma comunidade. Para tanto, envolve o abastecimento, a conservação e controle de qualidade, a segurança e a eficácia terapêutica, o acompanhamento e a avaliação da utilização, a obtenção e a difusão de informação sobre medicamentos e a educação permanente dos profissionais de saúde, do paciente e da comunidade para assegurar seu uso racional. ${ }^{2}$

Explorando mais a gestão da assistência farmacêutica, Perini ${ }^{3}$ argumenta que se destaca no interior do sistema de atenção à saúde, "um conjunto de tecnologias que têm por objetivo garantir práticas de prevenção, recuperação e alívio das doenças com base no uso ou em formas de evitar o uso de medicamentos". Ainda segundo o autor, este conjunto se caracteriza pela organização integrada de muitas tecnologias, com o propósito de garantir condições para o pleno exercício da relação entre prescrição, dispensação e uso de medicamentos. Assim, a assistência farmacêutica seria assumida como um sistema de tecnologias farmacêuticas, dividido em oito subsistemas: seleção, produção, programação, aquisição, armazenamento, distribuição, prescrição e dispensação.

Em relação ao que se entende por gestão da assistência farmacêutica, é importante destacar que o conceito de gestão por si só assume variações de acordo com as diferentes áreas de conhecimento. Um dos chamados conceitos-guia de gestão, parte do princípio de que a capacidade de gestão de uma organização pode ser avaliada por sua capacidade de formular políticas, de mobilizar recursos políticos e de disponibilizar recursos técnicos e administrativos para o alcance dos objetivos. ${ }^{4}$

Ao se considerar os oito subsistemas da assistência farmacêutica referidos anteriormente, bem como a dimensão governança do conceito de gestão ora apresentado, assume-se que a gestão da assistência farmacêutica em parte está relacionada à capacidade de alocar e gerenciar recursos técnicos e administrativos para assegurar o acesso e o uso racional de medicamentos.

Partindo desse ponto de vista, estudos que analisaram aspectos da gestão da assistência farmacêutica no SUS têm demonstrado deficiências que podem comprometer o alcance desse objetivo, criando limitações à garantia por parte do Estado do direito à saúde à população brasileira.

Entre eles, cita-se uma avaliação da assistência farmacêutica realizada pelo Ministério da Saúde, em parceria com a Organização Pan-Americana da Saúde (OPAS), que mostrou que a disponibilidade em estoque dos medicamentos principais foi de $73 \%$ nas unidades de saúde, $76 \%$ 
nas centrais de abastecimento farmacêutico municipais (CAF-M) e 77\% nas estaduais (CAF-E). O tempo de desabastecimento dos medicamentos principais, medido por meio de fichas de controle de estoque, foi em média de 84 dias nas unidades de saúde, 74 dias nas CAF-M e 128 dias nas CAF-E. Esse resultado mostrou que mesmo os medicamentos considerados imprescindíveis não estavam disponíveis nos serviços. ${ }^{5}$

Em relatório de pesquisa feita sobre a governança no SUS, o Banco Mundial afirma que o gerenciamento da logística de medicamentos absorve cerca de $20 \%$ dos recursos financeiros da saúde, apontando que há deficiência na qualidade dos serviços, que é atribuída a problemas de gestão e à ineficiência no uso dos recursos. ${ }^{6}$

Considerando que a maioria dos estudos publicados até o momento ou se referem à gestão da saúde como um todo ou têm amostra muito reduzida de municípios, torna-se relevante produzir mais evidências sobre problemas que podem comprometer o acesso a medicamentos por parte dos cidadãos. Nesse contexto, este artigo tem por objetivos descrever e analisar a gestão da assistência farmacêutica em alguns municípios brasileiros.

\section{MÉTODOS}

Foi conduzida pesquisa exploratória, transversal, elaborando-se questionário com questões fechadas sobre alguns aspectos da gestão municipal da assistência farmacêutica.

Os aspectos analisados estão relacionados à capacidade do município de alocar e gerenciar recursos técnicos, financeiros e administrativos a fim de assegurar o acesso e o uso racional de medicamentos. Nesse sentido, as categorias e aspectos empregados para nortear a avaliação foram:

a) institucionalização da assistência farmacêutica: formalização da assistência farmacêutica na estrutura organizacional da secretaria de saúde, existência de pelo menos um farmacêutico na coordenação de assistência farmacêutica, quando esta existe formalmente, ou trabalhando no nível central da secretaria de saúde com o tema assistência farmacêutica;

b) disponibilidade de serviços no município para atendimento de média e alta complexidade: dispensação de medicamentos no âmbito da saúde mental, de medicamentos do Componente Especializado da Assistência Farmacêutica, de medicamentos para atendimento em DST/Aids e disponibilidade no município de serviço próprio ou credenciado pelo SUS para atendimento em oncologia;

c) seleção de medicamentos: existência de lista de medicamentos para dispensação à população, atualização da lista nos últimos 5 anos, existência de comissão oficial que seleciona os medicamentos para a lista do município e seleção adequada de medicamentos nos municípios que informaram ter comissão oficial para esta finalidade; 
d) armazenamento: controle de estoque adequado em Unidades Básicas de Saúde - UBS;

e) dispensação: se há retenção de uma via da receita mesmo de medicamentos não controlados e se não há retenção da receita, se existe algum mecanismo de registro dos medicamentos dispensados;

f) ações judiciais: se a secretaria de saúde está cumprindo determinações judiciais para fornecimento de medicamentos.

Os parâmetros de adequabilidade para as categorias e aspectos foram baseados nos trabalhos desenvolvidos pela organização internacional Management Sciences for Health ${ }^{7}$ e por Marín et al. ${ }^{8}$

Para autorizar a participação do município e o envio dos dados, elaborou-se um Termo de Consentimento Livre e Esclarecido (TCLE), a ser assinado pelo secretário de saúde ou pelo profissional por ele designado, esclarecendo que não haveria na fase de divulgação da pesquisa identificação do município ou de qualquer profissional que tenha prestado as informações.

O questionário foi desenvolvido em formato eletrônico e ficou disponível na Internet durante todo o período da coleta de dados (julho a setembro de 2010). O acesso ao questionário foi feito mediante o preenchimento de nome de usuário e de senha que foram previamente encaminhados.

O tamanho da amostra foi definido em 431 municípios (Secretarias Municipais de Saúde SMS), considerando para o cálculo os seguintes parâmetros: a) erro de até $\%$ para que a gestão da assistência farmacêutica por parte da Secretaria de Saúde seja considerada satisfatória (ou seja, $\mathrm{p}=$ 0,02); b) nível de confiança de 95\%, o que corresponde ao índice de confiabilidade de 6,30 (Ci, $\alpha=$ 6,30 ); c) acréscimo de $40 \%$ ao valor da amostra (n) a título de possíveis perdas. Logo, $\mathrm{n}=\mathrm{Ci}, \alpha / \mathrm{p}$ $=6,3 / 0,02=315+40 \%$ de $315=431 .{ }^{9}$

A definição dos municípios da amostra foi feita por amostragem aleatória estratificada. Foram estabelecidos 7 estratos de acordo com o tamanho da população dos municípios. A definição do tamanho da amostra de cada estrato foi calculada pela ponderação da quantidade de municípios em cada estrato em relação ao total de municípios brasileiros. ${ }^{10}$ Assim, o tamanho da amostra em cada estrato foi assim distribuído: a) até 5.000 habitantes = 106 municípios; b) de 5.001 a $10.000=100$; c) de 10.001 a $20.000=100$; d) de 20.001 a $50.000=80$; e) de 50.001 a $100.000=24$; f) de 100.001 a $500.000=18 ; \mathrm{g}$ ) mais de $500.000=3$. Os municípios de cada estrato foram definidos por meio de amostragem sistemática.

Os contatos das secretarias de saúde foram obtidos de mala direta fornecida pelo Conselho Nacional de Secretarias Municipais de Saúde (CONASEMS). Foi enviada correspondência convencional para cada secretário de saúde dos municípios da amostra, contendo, carta-convite para participação na pesquisa, Termo de Consentimento Livre e Esclarecido - TCLE em duas vias, informação sobre o nome do usuário e senha para ter acesso ao questionário eletrônico, além de envelope com o porte pago para devolução do TCLE. 
Ao mesmo tempo, encaminhou-se mensagem eletrônica para todos os municípios da amostra que continham informação de correio eletrônico na mala direta do CONASEMS. Como muitas mensagens retornaram e alguns municípios não constavam com endereço de correio eletrônico, atualizou-se e completou-se este campo, por meio de pesquisa nos sítios eletrônicos dos Conselhos de Secretarias Municipais de Saúde (COSEMS), das prefeituras, secretarias de saúde e do Cadastro Nacional de Estabelecimentos de Saúde (CNES). Mensagens convidando à participação na pesquisa foram enviadas em mais quatro oportunidades, inclusive em um delas por membro do CONASEMS, encorajando os municípios a responderem o questionário. O convite também foi feito por fax para 209 municípios da amostra, os quais estavam com seus números atualizados no banco de contatos.

A realização da pesquisa também foi divulgada no sítio eletrônico do CONASEMS, no formato de notícia, ressaltando a importância da participação dos municípios e orientando sobre o acesso ao questionário.

Uma vez que alguns municípios relataram dificuldades no preenchimento do questionário na Internet, versão eletrônica dele (em editor de texto Word ${ }^{\circledR}$ ), para preenchimento e posterior envio por correio eletrônico, foi disponibilizada tanto por meio de mensagem eletrônica quanto na página de acesso ao questionário na Internet.

Foi criado um banco de dados a partir dos dados informados no sistema disponível no sítio eletrônico do questionário, o qual possibilitou rápida tabulação e análise exploratória, comparandose as respostas dadas pelas secretarias de saúde a parâmetros nacionais e internacionais de qualidade da gestão da assistência farmacêutica, tais como, existência de farmacêuticos na dispensação de medicamentos e mecanismos para controle de estoque.

A fim de verificar os valores alocados pelos municípios para a aquisição de medicamentos em 2009, foi feito levantamento de dados no sítio eletrônico do Sistema de Informação sobre Orçamentos Públicos em Saúde (SIOPS), em 16/8/2010, para as classificações contábeis relacionadas a estas despesas. O caminho da busca segue (http://siops.datasus.gov.br): Município>Dados informados> Consulta de um ou mais códigos contábeis, por categoria econômica, para um muncípio, por ano ou por série histórica $>$ Ano $=2009>$ Período $=$ Anual $>U$ UF $=$ Selecionar a UF, Município $=$ Selecionar 0 município, Tipo $=$ Despesa, Pasta $=$ Despesa Administração Direta - Saúde e Despesa Administração Indireta - Saúde, Fase $=$ Despesa Liquidada , Classificação Contábil = 3.3.3.90.30.00.01 - Aplicações diretas medicamentos, 3.3.3.90.32.03.01 - Aplicações diretas materiais de distribuição gratuita (medicamentos) e 3.3.3.40.00.00.01 - Transferência a municípios. A soma dos valores alocados nestas três classificações contábeis corresponde ao gasto total do município com medicamentos.

O gasto por habitante dos municípios com medicamentos foi obtido pela divisão do gasto total com medicamentos pela respectiva população, estimada para $1^{\circ}$ de julho de 2009 pelo IBGE. ${ }^{10}$ 
Também foram obtidos no sítio eletrônico do SIOPS indicadores sobre despesas com saúde, a fim de avaliar a situação dos municípios em relação a esta questão em 2009: a) participação da receita própria aplicada em saúde conforme a Emenda Constitucional no 29/2000 (\%); ${ }^{11}$ b) participação da despesa com medicamentos na despesa total com saúde (\%) e c) despesa total com saúde, sob a responsabilidade do município por habitante (R\$). O caminho segue: Município $>$ Indicadores $>$ Consultas - Indicadores Municipais $>$ Ano $=2009$, Período $=$ Anual, UF $=$ Selecionar a UF, Município = Selecionar o município.

Fez-se análise exploratória dos dados. Elaboraram-se tabelas e gráfico para facilitar a visualização dos resultados.

A pesquisa foi aprovada pelo Comitê de Ética em Pesquisa da Universidade Federal de São Paulo.

\section{RESULTADOS}

A taxa de resposta ao questionário da pesquisa foi de $8,4 \%$, ou seja, 36 respondentes entre os 431 municípios estratificados pelo tamanho de sua população. Os 36 municípios que responderam pertencem a 13 estados da federação, incluindo o Distrito Federal.

Destes, 12 são da Região Sudeste (33,3\%), 12 da Região Sul (33,3\%), 3 da Centro-Oeste (8,3\%), 7 da Nordeste $(19,4 \%)$ e 2 da Região Norte $(5,6 \%)$. Em relação ao tamanho da população dos municípios, 11 têm até 5.000 habitantes (30,6\%), 6 entre 5.001 e $10.000(16,7 \%), 6$ entre 10.001 e $20.000(16,7 \%), 6$ entre 20.001 e $50.000(16,7 \%), 2$ entre 50.001 e $100.000(5,6 \%), 2$ entre 100.001 e $500.000(5,6 \%)$ e 3 mais de 500.000 habitantes $(8,3 \%)$.

A tabela 1 apresenta os indicadores de despesas com saúde dos municípios que responderam o questionário da pesquisa.

Verificou-se que todos os municípios aplicaram em despesas com ações e serviços públicos de saúde mais que o mínimo obrigatório estabelecido pela Emenda Constitucional no 29/2000, ou seja, $15 \%$ da receita própria. ${ }^{11}$ Destaca-se, neste aspecto, que 3 municípios aplicaram mais de $27 \%$.

Tabela 1. Indicadores de despesas com saúde em 2009 de 36 municípios brasileiros que responderam o questionário da pesquisa.

\begin{tabular}{lccccc}
\hline $\begin{array}{c}\text { População } \\
\text { (habitantes) }\end{array}$ & $\begin{array}{c}\mathbf{N}^{0} \text { de } \\
\text { municípios }\end{array}$ & $\begin{array}{c}\text { Despesa total } \\
\text { média com } \\
\text { saúde, sob a } \\
\text { responsabilidade } \\
\text { do município, } \\
\text { por habitante } \\
\text { (R\$) }\end{array}$ & $\begin{array}{c}\text { Participação } \\
\text { média da } \\
\text { receita própria } \\
\text { aplicada em } \\
\text { saúde conforme } \\
\text { a EC 29/2000 } \\
\mathbf{( \% )}\end{array}$ & $\begin{array}{c}\text { Participação } \\
\text { média da } \\
\text { despesa com } \\
\text { medicamentos } \\
\text { na despesa } \\
\text { total com saúde } \\
\text { (\%) }\end{array}$ & $\begin{array}{c}\text { Gasto médio com } \\
\text { medicamentos } \\
\text { por habitante } \\
\text { (R\$) }\end{array}$ \\
\hline Até 5.000 & 11 & 485,91 & 18,87 & 6,55 & 32,06 \\
De 5.001 a 10.000 & 6 & 255,67 & 18,36 & 4,82 & 10,93
\end{tabular}




\begin{tabular}{lllllr} 
De 10.001 a 20.000 & 6 & 231,59 & 18,85 & 6,81 & 15,77 \\
De 20.001 a 50.000 & 6 & 263,64 & 23,14 & 3,73 & 9,62 \\
De 50.001 a 100.000 & 2 & 272,81 & 22,61 & 2,58 & 7,16 \\
De 100.001 a 500.000 & 2 & 342,63 & 20,82 & 3,21 & 10,31 \\
Mais de 500.000 & 3 & 415,50 & 21,79 & 5,08 & 26,38 \\
\hline
\end{tabular}

Elaboração própria com base em dados do Sistema de Informações sobre Orçamentos Públicos em Saúde - SIOPS e do Instituto Brasileiro de Geografia e Estatística - IBGE.

Em relação à participação média da despesa com medicamentos na despesa total com saúde, em termos percentuais, observa-se grande variação entre os municípios estratificados pelo tamanho de sua população. O mesmo se constata em relação ao gasto médio com medicamentos por habitante em 2009. Dos 36 municípios, 11 tiveram gasto acima da média geral ( $\mathrm{R} \$ 19,02)$, dos quais 1 tem população superior a 500 mil habitantes, enquanto os demais têm população inferior a 13 mil habitantes. Três municípios apresentaram gasto inferior à $\mathrm{R} \$ 1,75$ por habitante.

Quanto aos aspectos relativos à gestão da assistência farmacêutica, a tabela 2 resume os resultados. Nota-se que $44,4 \%(n=16)$ informaram ter a assistência farmacêutica formalizada, ou seja, prevista no organograma formal da secretaria de saúde. Destas, 93,4\% $(n=15)$ contam com pelo menos um farmacêutico na equipe. Nos casos em que esta coordenação não está formalizada $(\mathrm{n}=20)$, em 18 municípios (90\%) há pelo menos um farmacêutico trabalhando no nível central da secretaria de saúde com assistência farmacêutica.

Verificou-se que apenas um município não dispensa medicamentos para saúde mental. A dispensação de medicamentos do Componente Especializado da Assistência Farmacêutica ocorre em 16 municípios $(44,4 \%)$ e de medicamentos para atendimento em DST/Aids em 11 municípios (30,6\%). A menor disponibilidade de serviço de média e alta complexidade, entre os analisados, é do atendimento em oncologia, em que apenas 4 municípios $(11,1 \%)$ oferecem serviço próprio ou credenciado ao SUS.

Tabela 2. Aspectos relativos à gestão da assistência farmacêutica de 36 municípios brasileiros em 2010.

\begin{tabular}{|c|c|c|c|c|c|c|}
\hline \multirow[t]{2}{*}{ Categorias } & \multirow[t]{2}{*}{$\begin{array}{l}\text { Aspectos da gestão da assistência } \\
\text { farmacêutica }\end{array}$} & \multirow[b]{2}{*}{$\begin{array}{c}\text { Total de } \\
\text { Municípios }\end{array}$} & \multicolumn{2}{|c|}{ Sim } & \multicolumn{2}{|c|}{ Não } \\
\hline & & & $\mathbf{N}^{\mathbf{o}}$ & $\%$ & $N^{o}$ & $\%$ \\
\hline \multirow{3}{*}{$\begin{array}{l}\text { Institucionalização } \\
\text { da assistência } \\
\text { farmacêutica }\end{array}$} & $\begin{array}{l}\text { Formalização da assistência } \\
\text { farmacêutica na estrutura organizacional } \\
\text { da Secretaria de Saúde }\end{array}$ & 36 & 16 & 44,4 & 20 & 55,6 \\
\hline & $\begin{array}{l}\text { Trabalha pelo menos um farmacêutico } \\
\text { na coordenação de assistência } \\
\text { farmacêutica quando esta existe } \\
\text { formalmente }\end{array}$ & 16 & 15 & 93,8 & 1 & 6,3 \\
\hline & $\begin{array}{l}\text { Se não existe coordenação formal de } \\
\text { assistência farmacêutica, há pelo menos } \\
\text { um farmacêutico trabalhando no nível } \\
\text { central da secretaria de saúde }\end{array}$ & 20 & 18 & 90,0 & 2 & 10,0 \\
\hline
\end{tabular}




\begin{tabular}{|c|c|c|c|c|c|c|}
\hline \multirow{4}{*}{$\begin{array}{l}\text { Disponibilidade } \\
\text { de serviços no } \\
\text { município para } \\
\text { atendimento } \\
\text { de média e alta } \\
\text { complexidade }\end{array}$} & $\begin{array}{l}\text { Dispensação de medicamentos no } \\
\text { âmbito da saúde mental }\end{array}$ & 36 & 35 & 97,2 & 1 & 2,8 \\
\hline & $\begin{array}{l}\text { Dispensação de medicamentos } \\
\text { do Componente Especializado da } \\
\text { Assistência Farmacêutica }\end{array}$ & 36 & 16 & 44,4 & 20 & 55,6 \\
\hline & $\begin{array}{l}\text { Disponibilidade no município de serviço } \\
\text { próprio ou credenciado pelo SUS para } \\
\text { atendimento em oncologia }\end{array}$ & 36 & 4 & 11,1 & 32 & 88,9 \\
\hline & $\begin{array}{l}\text { Dispensação de medicamentos para } \\
\text { atendimento em DST/Aids }\end{array}$ & 36 & 11 & 30,6 & 25 & 69,4 \\
\hline \multirow{4}{*}{$\begin{array}{l}\text { Seleção de } \\
\text { medicamentos }\end{array}$} & $\begin{array}{l}\text { Existência de lista de medicamentos } \\
\text { para dispensação à população }\end{array}$ & 36 & 35 & 97,2 & 1 & 2,8 \\
\hline & Atualização da lista nos últimos 5 anos & 35 & 34 & 97,1 & 1 & 2,9 \\
\hline & $\begin{array}{l}\text { seleciona os medicamentos para a lista } \\
\text { do município }\end{array}$ & 35 & 20 & 57,1 & 15 & 42,9 \\
\hline & $\begin{array}{l}\text { Seleção adequada de medicamentos } \\
\text { nos municípios que informaram ter } \\
\text { comissão oficial para esta finalidade }\end{array}$ & 20 & 18 & 90,0 & 2 & 10,0 \\
\hline Armazenamento & $\begin{array}{l}\text { Controle de estoque adequado em } \\
\text { Unidades Básicas de Saúde - UBS }\end{array}$ & 36 & 26 & 72,2 & 10 & 27,8 \\
\hline \multirow[b]{2}{*}{ Dispensação } & $\begin{array}{l}\text { Há retenção de uma via da receita } \\
\text { mesmo de medicamentos não } \\
\text { controlados }\end{array}$ & 36 & 24 & 66,7 & 12 & 33,3 \\
\hline & $\begin{array}{l}\text { Se não, há retenção da receita, existe } \\
\text { algum mecanismo de registro dos } \\
\text { medicamentos dispensados }\end{array}$ & 12 & 12 & 100,0 & - & - \\
\hline Ações judiciais & $\begin{array}{l}\text { A secretaria de saúde está cumprindo } \\
\text { determinações judiciais para } \\
\text { fornecimento de medicamentos }\end{array}$ & 36 & 14 & 38,9 & 22 & 61,1 \\
\hline
\end{tabular}

Elaboração própria com base nas respostas dos municípios.

Em análise sobre a seleção de medicamentos, observou-se que 97\% $(\mathrm{n}=35)$ dos municípios informaram ter lista de medicamentos para dispensação à população e, destes, 34 disseram que foi atualizada nos últimos 5 anos. Mas somente 20 municípios $(57,1 \%)$ responderam que possuem comissão oficial para a seleção de medicamentos, sendo que destes, o processo de trabalho foi considerado adequado para 18 deles (90\%).

No caso do controle de estoque, avaliou-se como adequado o processo realizado por 26 municípios $(72,2 \%)$. Na dispensação de medicamentos, 24 municípios $(66,7 \%)$ retêm uma via da receita médica, mesmo para medicamentos não controlados (não sujeitos ao controle especial). Os demais responderam que dispõem de outros mecanismos de registro dos medicamentos dispensados para cada paciente $(\mathrm{n}=12)$.

Em relação às ações judiciais, dos 36 municípios 14 (38,9\%) informaram estarem cumprindo determinação judicial para o fornecimento de medicamentos, sendo que 4 percentem ao estado de São Paulo, 3 de Minas Gerais, 1 de Goiás, 1 do Paraná, 3 do Rio Grande do Sul, 1 de Santa Catarina 
e 1 do Distrito Federal. Quando classificados por tamanho de sua população, tem-se que: 1 possui até 5.000 habitantes, 3 entre 5.001 e 10.000, 2 entre 10.001 e 20.000, 3 entre 20.001 e 50.000, 1 entre 50.001 e $100.000,2$ entre 100.001 e 500.000 e 2 com mais de 500.000 habitantes.

A tabela 3 apresenta os resultados para o número de Unidades Básicas de Saúde - UBS que dispensam medicamentos e que possuem farmacêutico. Das 564 UBS que dispensam medicamentos, $14,36 \%$ possuem farmacêuticos em regime de dedicação exclusiva e 93,06\% dispensam medicamentos controlados e possuem pelo menos um farmacêutico.

Tabela 3. Disponibilidade de farmacêuticos em Unidades Básicas de Saúde (UBS) que dispensam medicamentos de 36 municípios brasileiros em 2010.

\begin{tabular}{|c|c|c|}
\hline Categorias & $\mathbf{N}^{\mathbf{o}}$ & $\%$ \\
\hline UBS que dispensam medicamentos & 564 & - \\
\hline UBS com farmacêuticos em dedicação exclusiva & 81 & $14,36 \%$ \\
\hline UBS que dispensam medicamentos controlados & 72 & $12,77 \%$ \\
\hline UBS que dispensam medicamentos controlados e possuem pelo menos um fa & 67 & $93,06 \%$ \\
\hline
\end{tabular}

Elaboração própria com base nas respostas dos municípios.

Observou-se que em 13 municípios (36\%) todas as UBS contam com farmacêutico em dedicação exclusiva. Dos 36 municípios, 26 dispensam medicamentos controlados em UBS. Apenas dois municípios informaram dispensar controlados em UBS e não dispor de farmacêutico.

Quanto à distância a ser percorrida para atendimento em serviços de média e alta complexidade em saúde, o gráfico 1 apresenta os resultados.

Observa-se que para a dispensação de medicamentos do Componente Especializado da Assistência Farmacêutica os pacientes oriundos de uma população maior que 86 mil pessoas têm de percorrer entre 50 e $99 \mathrm{~km}$ e de uma população maior de 64 mil pessoas mais de $150 \mathrm{~km}$ para a dispensação dos medicamentos. No caso de atendimento em DST/Aids, os pacientes oriundos de uma população maior que 136 mil pessoas percorrem mais de $150 \mathrm{~km}$. Na oncologia, os pacientes de uma população maior que 464 mil pessoas percorrem entre 50 e $99 \mathrm{~km}$ e de uma população maior que 215 mil pessoas mais de $150 \mathrm{~km}$.

Em relação ao encaminhamento de pacientes para serviço de referência em média e alta complexidade em saúde, fora do município e independentemente do tipo de serviço, 33 municípios $(91,7 \%)$ informaram referenciá-los. Destes, 8 (24,2\%) disseram que quando os pacientes retornam ao município de origem conseguem atender às prescrições que eles trazem porque dispõem de medicamentos especializados em sua lista de medicamentos; 7 municípios $(21,2 \%)$ informaram que nesta situação compram os medicamentos por meio da secretaria de saúde; 2 municípios $(6,1 \%)$ 
Gráfico 1. População de 36 municípios brasileiros e distância a ser percorrida para dispensação de medicamentos ou tratamento e serviços de média e alta complexidade em 2010.

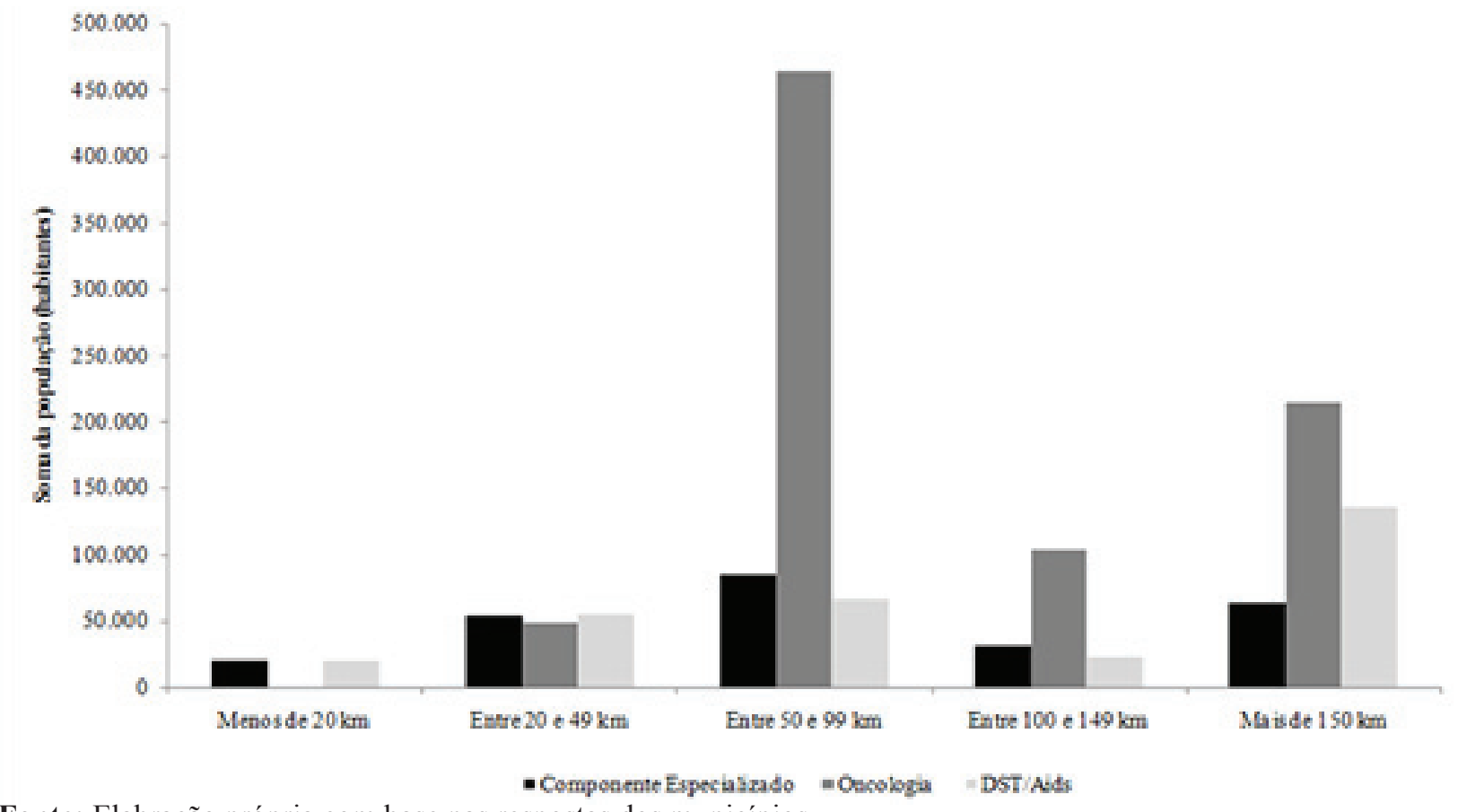

Fonte: Elabração própria com base nas respostas dos municípios.

que os medicamentos não são comprados nem pela secretaria de saúde e nem pela assistência social do município e 16 municípios (48,5\%) informaram que, no caso de pacientes que não podem comprar os medicamentos, são encaminhados para o serviço de assistência social para que este serviço os compre e forneça.

Dos 36 municípios, $15(41,7 \%)$ respondentes classificaram como adequada a oferta de medicamentos à população, enquanto $21(58,3 \%)$ consideraram que há pontos a melhorar, seja quanto aos medicamentos sob responsabilidade do município $(\mathrm{n}=7,19 \%)$, quanto do estado e Ministério da Saúde ( $\mathrm{n}=14,38,9 \%)$.

\section{DISCUSSÃO}

Uma primeira questão que deve ser abordada é a baixa taxa de resposta $(8,4 \%)$ ao questionário. Apenas 36 dos 431 municípios participaram da pesquisa.

Vasconcelos et al, ${ }^{12}$ ao mapear as vantagens e limitações da utilização de questionário eletrônico por meio da Internet, citam algumas desvantagens deste método para coleta de dados: a) restrição dos respondentes às pessoas com acesso à Internet; b) impessoalidade e problemas de privacidade; c) dificuldades de incluir incentivos para envio da resposta; d) baixo índice de resposta, menores que todos os outros métodos de aplicação de questionário; e, por fim, e) baixa confiabilidade nos dados, uma vez que os respondentes podem prestar informações que não são passíveis de verificação. As vantagens consistem em: i) baixo custo; ii) agilidade para aplicação; iii) tabulação rápida dos dados, evitando-se possíveis erros de digitação; iv) facilidade de utilizar maiores amostras; v) 
flexibilidade e diversidade na elaboração de questões; e vi) exigência de resposta completa.

Ainda conforme Vasconcelos et al, ${ }^{12}$ pesquisa feita com o objetivo de analisar a utilização das tecnologias de informação por professores de universidade pública brasileira, por meio do envio de questionários em papel e via e-mail, constatou que a taxa de resposta dos questionários em papel (30\%) foi maior que dos questionários via e-mail $(8,2 \%)$. Em outro trabalho, os pesquisadores enviaram um questionário em papel para professores de uma universidade pública, com a opção de preenchimento em papel ou através de uma página na Internet. Dos 102 professores que receberam o questionário, 65 responderam, dos quais $64,6 \%$ utilizaram o questionário em papel e 35,4\% responderam por meio da página na Internet.

Mesmo que as taxas de resposta sejam baixas para pesquisas que utilizam como meio para a coleta de dados o questionário eletrônico, acreditava-se obter maior índice de resposta em virtude das estratégias adotadas: a) obtenção de apoio institucional do Conselho Nacional de Secretarias Municipais de Saúde (CONASEMS), que inclusive divulgou a realização da pesquisa em seu sítio eletrônico; b) envio de mensagem eletrônica por membro do CONASEMS, estimulando os municípios a participarem da pesquisa; c) envio de correspondência convencional (correio) convidando à participação, com porte pago para devolução do Termo de Consentimento Livre e Esclarecido - TCLE; e d) envio de fax para quase 50\% dos municípios da amostra.

A comunicação entre secretarias municipais e estaduais de saúde, e mesmo com o Ministério da Saúde, tem se dado com o uso da Internet para vários sistemas de informação, de forma que o acesso à rede mundial de computadores não deve ter sido o fator principal de constrangimento às respostas. Também é evidência disso o fato de que a maioria dos municípios da amostra continha endereço de correio eletrônico no banco de contatos do CONASEMS e, ainda quando este não constava, estava incorreto ou desatualizado, foi possível completá-lo e substituí-lo em consulta aos sítios eletrônicos dos Conselhos de Secretarias Municipais de Saúde - COSEMS e do Cadastro Nacional de Estabelecimentos de Saúde - CNES.

Fatores como o tamanho do questionário e a natureza das perguntas podem ter inibido a participação na pesquisa. Neste último caso, em virtude de sentimento de exposição dos problemas quanto à gestão da assistência farmacêutica no município, mesmo sendo garantido o anonimato dos participantes quando da divulgação dos resultados.

De todo modo, embora não se tenha conseguido representatividade em relação aos municípios brasileiros, os resultados obtidos são importantes porque se referem a alguns municípios, de tamanho de população diferenciado (todos os estratos criados para análise neste trabalho), os quais vivenciam o contexto da gestão da assistência farmacêutica em seus estados e regiões, sob uma diretriz nacional. Se o viés de participação for considerado, pode-se estar trabalhando com os mais organizados ou mais interessados, então este trabalho pode apresentar resultados considerados mais positivos em termos de gestão que aqueles para a maioria dos municípios brasileiros. 
Na tabela 1 foram apresentados indicadores de despesas com saúde dos municípios, verificandose que todos eles aplicaram em despesas com ações e serviços públicos de saúde mais que o mínimo obrigatório estabelecido pela Emenda Constitucional n ${ }^{\circ}$ 29/2000 em 2009. ${ }^{11}$ Isso significa que todos estão cumprindo seu dever em relação ao financiamento da saúde, de acordo com os preceitos legais, o que não implica em suficiência de recursos financeiros. É possível notar que a despesa total com saúde por habitante variou muito entre os municípios, o que sugere oferta diferenciada de ações e serviços à população, bem como capacidade alocativa diversificada. Além disso, o SUS é considerado subfinanciado. Em 2006, por exemplo, as despesas com ações e serviços públicos de saúde totalizaram apenas 3,55\% do Produto Interno Bruto - PIB brasileiro, ${ }^{13}$ percentual bem abaixo daquele de países desenvolvidos que têm sistemas universais de saúde como França (11,1\%), Reino Unido $(8,4 \%)$ e Canadá $(10 \%) .{ }^{14}$

Em relação ao gasto com medicamentos, grande variação também é observada, parecendo haver uma tendência a que municípios menores tenham maior gasto médio. Considerando que em termos qualitativos as listas de medicamentos variam pouco entre os municípios devido ao financiamento federal da assistência farmacêutica na atenção básica e da indução para aquisição de medicamentos da Relação Nacional de Medicamentos Essenciais - Rename, ${ }^{15}$ além do fato de que a maioria dos municípios teve gasto abaixo da média $(\mathrm{R} \$ 19,02)$ e que dos 11 que tiveram gasto acima da média, 10 tinham população inferior a 13 mil habitantes, parece que questões econômicas como menor poder de compra, em virtude de aquisição em pequena quantidade e baixa possibilidade de negociação, estão contribuindo para maior gasto por habitante. Neste trabalho, não é possível fechar conclusão a este respeito em virtude da falta de representatividade da amostra. Entretanto, trabalho recente já comprovou esta situação, mostrando que o gasto dos municípios com população abaixo de 10 mil habitantes chega a ser quase 4 vezes aquele dos municípios com mais de 500 mil habitantes. ${ }^{16}$

O que se pode dizer ainda sobre o financiamento de medicamentos no SUS é que, assim como o que ocorre com o sistema como um todo, os recursos financeiros são insuficientes. Dados da Pesquisa Nacional por Amostra de Domicílios (PNAD) em 2008 demonstram que 48,9\% dos entrevistados que precisaram de medicamentos na última vez, não obtiveram nenhum deles gratuitamente. ${ }^{17}$ Além disso, em 2007, de acordo com a conta-satélite de saúde, as famílias financiaram 90\% do consumo final de medicamentos enquanto a Administração Pública participou com aproximadamente $10 \% .{ }^{18} \mathrm{O}$ consumo final se refere aos medicamentos que são utilizados ambulatorialmente, fora das unidades de saúde.

Quanto aos aspectos relativos à gestão da assistência farmacêutica, resumidos na tabela 2 , o achado de que $44 \%$ têm a assistência farmacêutica formalizada é igual ao encontrado em avaliação realizada em 18 municípios no estado de Minas Gerais. ${ }^{19}$ Mas o que se verifica é que ainda há problemas para a atuação das coordenações de assistência farmacêutica no SUS, tais como, constrangimento na sua autonomia, não funcionamento da Comissão de Farmácia e Terapêutica - CFT e ausência do farmacêutico em atividades como a dispensação e programação de medicamentos. ${ }^{20}$ 
A instituição Management Sciences for Health, em colaboração com a OMS, alerta para o fato de que embora a descentralização da assistência farmacêutica traga benefícios por propiciar maior envolvimento local, maior responsabilização dos gestores, aumentar a flexibilidade para ajustes locais, facilitar a comunicação e implementar mais rapidamente mudanças, pode acarretar problemas que comprometem o acesso a medicamentos como, por exemplo, a não disponibilidade de pessoal capaz para gerenciar os serviços farmacêuticos. ${ }^{7}$

Para os municípios desta pesquisa, a disponibilidade de farmacêuticos no nível central da secretaria de saúde não parece ser o problema principal, na medida em que, mesmo nos municípios em que a assistência farmacêutica não foi formalizada, há pelo menos um profissional trabalhando nesta área. Entretanto, o fato de dispor deste profissional não implica suficiência para o gerenciamento das ações e serviços de assistência farmacêutica, tanto no aspecto quantitativo quanto qualitativo.

No que se refere ao atendimento de média e alta complexidade em saúde, verificou-se que a maioria dos municípios referencia os pacientes para serviços fora do município, especialmente para quimioterapia e dispensação de medicamentos em DST/Aids. Isso pode ser explicado pelo tamanho dos municípios da amostra e pela lógica hierarquizada de organização do SUS, em que serviços mais complexos são oferecidos em municípios maiores, com maior capacidade instalada. Entretanto, chamou a atenção o fato de que um município não dispensa medicamentos no âmbito da saúde mental, o que pode dificultar o acesso dos pacientes ao tratamento.

Em relação à seleção de medicamentos, a maioria possui lista de medicamentos e a atualizou nos últimos 5 anos, conforme preconizado. ${ }^{7,8} \mathrm{O}$ problema está na falta de uma comissão oficial para a seleção, o que acontece em $43 \%$ dos municípios, e na seleção inadequada naqueles que possuem comissão (10\% deles). Considerou-se inadequada a seleção por qualquer processo que não fosse feito por uma comissão oficial, criada para estudar literatura científica a partir de provas de eficácia, segurança e custo-efetividade dos medicamentos e/ou que selecionasse os medicamentos a partir das relações, nacional e/ou estadual, de medicamentos essenciais. ${ }^{7,8,21}$

A falta da comissão, em parte, pode ser explicada nos municípios muito pequenos que apenas ofertam à população os medicamentos da Rename, pactuados com a Secretaria de Estado da Saúde para a assistência farmacêutica na atenção básica.

De todo modo, ressalta-se que a seleção de medicamentos é processo fundamental em gestão da assistência farmacêutica. Os critérios empregados para a realização das escolhas são: necessidade, verificada por meio da avaliação do perfil epidemiológico da população-alvo, eficácia terapêutica, relação risco-benefício e relação custo-benefício. ${ }^{21}$

As vantagens de adoção de relação limitada de medicamentos são de várias ordens: 1) suprimentos: facilita a aquisição, o armazenamento e a distribuição; os estoques são menores e melhora a garantia da qualidade; a dispensação torna-se mais fácil; 2) prescrição: o treinamento 
pode ser focado e conseqüentemente mais fácil; propicia-se mais experiência com poucos fármacos; evitam-se as alternativas irracionais de tratamento; foca-se na informação sobre medicamentos; facilita o melhor reconhecimento de eventos adversos; 3) custo: os preços ficam mais baixos devido à concorrência no momento da compra; 4) uso pelos pacientes: foca o esforço na educação; reduz a confusão e aumenta a adesão ao tratamento; melhorando a disponibilidade de medicamentos. ${ }^{7}$

Quanto ao controle de estoque, 27,8\% ainda fazem controle inadequado. Foi considerado como inadequado qualquer processo que não garantisse monitoramento das entradas e saídas diárias dos medicamentos, seja por meio de fichas de prateleira, planilha eletrônica ou sistema informatizado. ${ }^{7,8,21}$ Estudo publicado em 2008, o qual analisou amostra de 10,7\% dos municípios brasileiros $(\mathrm{n}=597)$, encontrou que em $71 \%$ dos municípios ou faltava mecanismo para controle de estoque ou este era deficiente. ${ }^{22}$

Todos os municípios informaram dispor de instrumentos para registro da dispensação aos pacientes, seja por retenção de uma via da receita ou por qualquer outro meio, o que é relevante nas situações em que houver necessidade de rastrear os pacientes em tratamento.

Quanto às ações judiciais, verificou-se que os 14 municípios que estão cumprindo determinação judicial para fornecimento de medicamentos encontram-se no eixo centro-sul do país e que estão distribuídos por tamanho de população nas sete categorias definidas para a amostragem estratificada inicial, de forma que se conta com dados oriundos de municípios pequenos e grandes. Isso revela que o fenômeno não está restrito a grandes centros urbanos, mas está atingindo pequenos municípios, ao contrário do que se poderia imaginar, até mesmo porque as evidências produzidas em estudos publicados até o momento são advindas ou de Secretarias de Estado da Saúde ou de secretaria de saúde de grande município. ${ }^{23-26}$ Esta situação preocupa na medida em que em municípios menores é mais improvável que os gestores disponham de quadro de pessoal mais preparado para argumentar a pertinência ou não das solicitações de medicamentos nos processos. Tal situação pode contribuir para a escalada das demandas.

No tocante à presença de farmacêuticos em UBS (tabela 3), observa-se o esforço de alocálos especialmente em unidades que dispensam medicamentos controlados, o que já evidencia cuidado com a dispensação destes medicamentos, ainda que na legislação brasileira a exigência de sua presença exista para qualquer serviço que dispense medicamentos, não importando o tipo de medicamento dispensado. ${ }^{27}$ Entretanto, ainda são muitas as UBS que não possuem o profissional, especialmente em municípios maiores. Em avaliação da assistência farmacêutica de 20 municípios da região noroeste do estado do Rio Grande do Sul, encontrou-se que apenas 5 contavam com farmacêutico responsável pela farmácia. ${ }^{28}$

Rozenfeld ${ }^{29}$ defende que é fundamental que toda farmácia tenha um farmacêutico durante todo o horário de funcionamento e argumenta que em vários países seu papel é reconhecido na melhoria da qualidade da prescrição e da dispensação, o que possibilitou avanços maiores no uso 
de medicamentos.

No caso do SUS, há ainda um longo caminho para o alcance desta realidade. Muitas unidades de saúde ainda dispõem de profissionais não preparados para a dispensação de medicamentos em suas farmácias, considerando que a maioria das UBS não possui farmacêuticos.

Em relação às distâncias para atendimento de média e alta complexidade, verifica-se que vários pacientes precisam percorrer mais de $150 \mathrm{~km}$ para obter medicamentos do Componente Especializado da Assistência Farmacêutica, em DST/Aids e realizar quimioterapia. Embora o Ministério da Saúde assuma como aceitável que o paciente percorra até 3 horas, pelos meios normais de locomoção, para ter acesso a serviços de maior complexidade ambulatorial, ${ }^{30}$ tal situação parece não favorecer o acesso a estes tratamentos. Os pacientes podem ter dificuldade de locomoção, impossibilidade de se ausentar por períodos prolongados do trabalho e até mesmo insuficiência de recursos para realizar estes deslocamentos, com comprometimento do acesso, caso os municípios não providenciem transporte para estas situações.

No caso do encaminhamento para qualquer serviço de referência em média e alta complexidade em saúde, fora do município, há problemas para garantir o acesso aos medicamentos prescritos. Nas três situações identificadas, compra dos medicamentos pela secretaria de saúde (21\%), não fornecimento do medicamento (6\%) e encaminhamento para a assistência social (49\%), a integralidade da assistência e o acesso com uso racional ficam comprometidos. $\mathrm{O}$ acesso a medicamentos constitui um direito e não beneficência em favor dos mais pobres. Esta situação revela que ainda há lacunas a resolver em relação aos medicamentos utilizados em atendimentos de média e alta complexidade, o que implica a necessidade de discutir regionalização sob a perspectiva da assistência farmacêutica a fim de garantir integralidade da terapêutica.

Ainda assim, apesar das dificuldades apontadas, 42\% dos respondentes classificaram a assistência farmacêutica como adequada em seus municípios, o que sugere desconhecimento sobre os parâmetros de qualidade necessários.

\section{CONSIDERAÇÕES FINAIS}

Embora a assistência farmacêutica constitua parte fundamental da assistência à saúde, muito raramente tem sido considerada como tal, sendo contemplada com iniciativas de fortalecimento de sua gestão. Isso mostra que embora o SUS tenha se organizado para priorizar as ações em saúde e mais que isso, para selecionar os medicamentos utilizados para prestar o atendimento, considerando a eficácia, segurança e custo-efetividade desses produtos, ainda tem muito para avançar e estruturar a fim de garantir o acesso a medicamentos e a efetividade das ações de saúde por meio da assistência farmacêutica. 


\section{REFERÊNCIAS BIBLIOGRÁFICAS}

1. Organización Mundial de la Salud - OMS. Perspectivas políticas de medicamentos de la OMS. 4 - Selección de medicamentos esenciales. Ginebra, OMS, 2002.

2. Brasil, Ministério da Saúde. Política Nacional de Medicamentos. Brasília: Ministério da Saúde, 2001.

3. Perini E. Assistência farmacêutica: fundamentos teóricos e conceituais. In: Acurcio FA(Org). Medicamentos e assistência farmacêutica. Belo Horizonte: COOPMED, 2003.

4. Guimarães MCL, Chaves dos Santos SM, Melo C, Sanches Filho A. Avaliação da capacidade de gestão de organizações sociais: uma proposta metodológica em desenvolvimento. Cad. Saúde Pública 2004;20(6):1642-1650.

5. Ministério da Saúde, Organização Pan-Americana da Saúde (Opas). Avaliação da assistência farmacêutica no Brasil: estrutura, processo e resultados. Brasília: Ministério da Saúde/Opas, 2005.

6. Banco Mundial. Governança no Sistema Único de Saúde (SUS) do Brasil. Melhorando a Qualidade do Gasto Público e Gestão de Recursos. Washington: Banco Mundial; 2007.

7. Management Science for Health (MSH). In Collaboration with the World Health Organization. Managing Drug Supply. Washington: Kumarian Press, 1997.

8. Marín N, Luiza VL, Osório-de-Castro CGS, Machado-dos-Santos S. Assistência farmacêutica para gerentes municipais. Rio de Janeiro: OPAS/OMS, 2003.

9. Tribunal de Contas da União (TCU). Técnicas de amostragem para auditorias. Brasília: TCU, 2002.

10. Instituto Brasileiro de Geografia e Estatística - IBGE. Estimativas populacionais para os municípios brasileiros em 01.07.2009. Disponível em: http://www.ibge.gov.br/home/estatistica/ populacao/estimativa2009/default.shtm. Acessado em 4 de janeiro de 2012.

11. Brasil. Emenda Constitucional no 29, de 13 de setembro de 2000. Altera os arts. 34, 35, 156, 160, 167 e 198 da Constituição Federal e acrescenta artigo ao Ato das Disposições Constitucionais Transitórias, para assegurar os recursos mínimos para o financiamento das ações e serviços públicos de saúde. Disponível em: http://www.planalto.gov.br/ccivil_03/constituicao/emendas/emc/emc29. htm. Acessado em 4 de janeiro de 2012.

12. Vasconcelos L, Guedes LFA. E-Surveys: Vantagens e limitações dos questionários eletrônicos via Internet no contexto da pesquisa científica. X SEMEAD - Seminários em Administração FEAUSP, 9 e 10 de agosto de 2007. Disponível em: http://www.ead.fea.usp.br/Semead/10semead/ ISSN 1982-8829 Tempus, actas de saúde colet, Brasília, 8(4), 11-29, dez, 2014// 
sistema/resultado/trabalhosPDF/420.pdf. Acessado em 4 de janeiro de 2012.

13. Jorge EA. O SIOPS como instrumento de gestão. In: I Congresso Brasileiro de Política, Planejamento e Gestão em Saúde. Salvador, 24 a 26 de agosto de 2010. Disponível em: http:// portal.saude.gov.br/portal/arquivos/pdf/planejasus_seminario10_siops_eliasjorge.pdf. Acessado em 4 de janeiro de 2012.

14. World Health Organization - WHO. Countries. Statistics: Total expenditure on health as \% of GDP (2006). Disponível em: http://www.who.int/countries/en/\#B. Acessado em 4 de janeiro de 2012.

15. Ministério da Saúde. Relação Nacional de Medicamentos Essenciais - Rename. Brasília: Ministério da Saúde, 2010.

16. Vieira FS, Zucchi P. Aplicações diretas para aquisição de medicamentos no Sistema Único de Saúde. Rev. Saúde Pública 2011:45(5):906-913.

17. Instituto Brasileiro de Geografia e Estatística - IBGE. PNAD 2008 - Um panorama da saúde no Brasil - Microdados. Disponível em: http://www.ibge.gov.br/home/estatistica/populacao/ panorama_saude_brasil_2003_2008/microdados.shtm. Acessado em 4 de janeiro de 2012.

18. Instituto Brasileiro de Geografia e Estatística - IBGE. Conta-satélite de saúde do Brasil: 2005 - 2007. Rio de Janeiro: IBGE, 2009.

19. Moura CS, Perini E. Evaluation of pharmaceutical assistance in municipalities in the state of Minas Gerais. Brazilian Journal of Pharmaceutical Sciences 2009;45(2):279-286.

20. Barreto JL, Guimarães MCL. Avaliação da gestão descentralizada da assistência farmacêutica básica em municípios baianos, Brasil. Cad. Saúde Pública 2010;26(6):1207-1220.

21. Dupim JAA. Assistência farmacêutica - um modelo de organização. Belo Horizonte: Segrac, 1999.

22. Vieira FS. Qualificação dos serviços farmacêuticos no Brasil: aspectos inconclusos da agenda do Sistema Único de Saúde. Rev Panam Salud Publica 2008:24(2):91-100.

23. Messeder AM, Osório-de-Castro CGS, Luiza VL. Mandados judiciais como ferramenta para garantia do acesso a medicamentos no setor público: a experiência do Estado do Rio de Janeiro, Brasil. Cad Saúde Pública 2005;21:525-34.

24. Vieira FS. Distorções causadas pelas ações judiciais à política de medicamentos no Brasil. Rev. Saúde Pública 2007;41(2):214-222. 
25. Pereira JR, Santos RI, Nascimento-Junior JM, Schenkel PE. Análise das demandas judiciais para o fornecimento de medicamentos pela Secretaria de Estado da Saúde de Santa Catarina nos anos de 2003 e 2004. Ciência \& Saúde Coletiva 2010:15(supl. 3):3551-3560.

26. Pepe VLE, Ventura M, Sant'ana JMB, Figueiredo TA, Souza VR, Simas L et al. Caracterização de demandas judiciais de fornecimento de medicamentos "essenciais" no Estado do Rio de Janeiro, Brasil. Cad. Saúde Pública 2010;26(3):461-471.

27. Brasil. Lei $n^{0}$ 5.991, de 17 de dezembro de 1973. Dispõe sobre o controle sanitário do comércio de drogas, medicamentos, insumos farmacêuticos e correlatos, e dá outras providências. Disponível em: http://www.anvisa.gov.br/legis/consolidada/lei_5991_73.htm. Acessado em 4 de janeiro de 2012.

28. Bernardi CLB, Bieberbach EW, Thomé HI. Avaliação da assistência farmacêutica básica nos municípios de abrangência da $17^{\text {a }}$ Coordenadoria Regional de Saúde do Rio Grande do Sul. Saúde e Sociedade 2006;15(1):73-83.

29. Rozenfeld S. Farmacêutico: profissional de saúde e cidadão. Ciência \& Saúde Coletiva 2008;13(Sup):561-568.

30. Brasil, Ministério da Saúde. Parâmetros para as programações básicas de saúde. Brasília: Ministério da Saúde, 2001. Disponível em: http://www.opas.org.br/servico/arquivos/Sala5406.pdf. Acessado em 4 de janeiro de 2012.

Artigo apresentado em 03-02-14

Artigo aprovado em 22-09-14 Artigo publicado no sistema em 20-12-14 